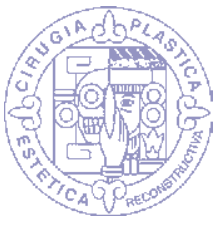

doi: $10.35366 / 91731$

https://dx.doi.org/10.35366/91731

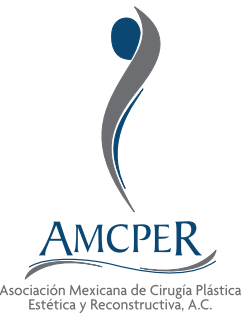

\title{
Reacciones adversas con el uso de rellenos faciales
}

\author{
Adverse reactions with the use of facial fillers
}

\section{Dr. Mario Israel Ortega-Ascanio,* Dra. Ana Karen Medina-Lira, ${ }^{\ddagger}$ Dra. Karina Lizbeth Lara-Sampayo ${ }^{\S}$}

\begin{abstract}
Palabras clave: Rellenos faciales, granuloma, efectos adversos, rellenos inyectables.

Keywords: Facial fillers, granuloma, adverse effects, injectable fillers.
\end{abstract}

${ }^{*}$ Cirujano plástico y reconstructivo del Hospital General de Castillo. Fellowship en cirugía reconstructiva y estética mamaria en España.

‡ Médico residente de Cirugía Plástica y Reconstructiva. Hospital Universitario «Dr. José Eleuterio González».

${ }^{\S}$ Médico cirujano y pasante en el Departamento de Cirugía Plástica y Reconstructiva. Hospital Universitario «Dr. José Eleuterio González».

Los autores de este artículo no tienen conflicto de intereses qué declarar.

Recibido:

26 septiembre 2019 Aceptado para publicar: 04 noviembre 2019
RESUMEN

El afán por el rejuvenecimiento facial de nuestra sociedad actual se remonta desde finales del siglo XIX, periodo en el que se empezaron a producir rellenos faciales para corregir o restaurar el volumen de los tejidos con fines terapéuticos y estéticos. El uso de estas sustancias para aumentar los tejidos blandos por motivos estéticos puede producir la aparición de granulomas, entre otros efectos indeseables. Las mejoras introducidas en esas sustancias han conseguido disminuir la incidencia de reacciones adversas, pero no su desaparición. La reacción granulomatosa a cuerpo extraño aparece después de un periodo de tiempo, el cual varía desde 5 meses hasta 15 años. El mecanismo de acción permanece desconocido, aunque se ha sugerido que una infección podría desencadenar una reacción inmunológica cruzada o que podría existir una estimulación de la inmunidad retardada. Presentamos el caso de una mujer en la sexta década de vida, quien presentó enfermedad por modelantes; mostramos los detalles del diagnóstico y tratamiento, este último llevado a cabo con esteroides administrados por vía sistémica y resección quirúrgica de la reacción granulomatosa.

\section{ABSTRACT}

The desire for facial rejuvenation of contemporary society dates back to the end of the 19th century, a period in which facial fillers began to be produced to correct or restore tissue volume for therapeutic and aesthetic purposes. The use of substances to increase soft tissues for aesthetic reasons can cause the appearance of granulomas to foreign bodies, among other undesirable effects. The improvements introduced in these substances have managed to reduce the incidence of adverse reactions, but not their disappearance. The granulomatous reaction to a foreign body appears after a variable period of time and ranges from 5 months to 15 years. The mechanism of action remains unknown, although it has been suggested that an infection could trigger a cross-immune reaction or that there may be delayed immunity stimulation. We present the case of a woman in the sixth decade of life who presented disease caused by modeling substances, the details of the diagnosis and treatment, the latter carried out with steroids administered systemically and surgical resection of the granulomatous reaction.

\section{INTRODUCCIÓN}

$E^{n}$ nuestra sociedad contemporánea, la avidez por el rejuvenecimiento facial se remonta hacia finales del siglo XIX, periodo en el que se comenzaron a producir rellenos faciales para corregir o restaurar con fines terapéuticos o estéticos el volumen de los tejidos. ${ }^{1,2}$

La pérdida de colágena tipo I en la dermis es la principal causa de las líneas de expresión; para su corrección, se requiere un relleno facial idóneo con una vida media larga. El mecanismo de acción es similar en todos ellos: se trata de materiales supuestamente inertes que, al ser introducidos en los tegumentos, producen un aumento de volumen per se y una infiltración progresiva de una matriz de colágena, que contribuye a su vez a ese aumento. ${ }^{3,4}$

La selección del material debe vislumbrar las características propias y su uso, además de tomar en cuenta otros factores inherentes al paciente que puedan desencadenar reacciones adversas, incluyendo hipersensibilidad, estado general y nutricional. ${ }^{5,6}$

Los efectos adversos de los rellenos faciales se pueden dividir en dos: tempranos, que se 
presentan con eritema, edema, dolor y prurito en el sitio de inyección, infección secundaria (evidenciada a menudo por pápulas y nódulos), necrosis tisular por oclusión vascular o cambios de coloración en la piel; y tardíos, con infecciones de predominio bacteriano, inflamación granulomatosa, la cual puede abarcar desde cambios histológicos subclínicos hasta nódulos desfiguradores, o necrosis local secundaria a oclusión arterial por inyección profunda. $^{7}$

\section{CASO CLÍNICO}

Se trata de una mujer de 60 años, sin antecedentes médicos de importancia (Figura 1), quien inició su padecimiento un año antes de su valoración al aplicarse rellenos faciales de origen desconocido en el surco nasogeniano, en la región periorbitaria, malar y frontal, y mejillas (Figura 2), lo que le produjo una reacción inflamatoria severa. Una semana después apareció un nódulo en la región frontal, de consistencia firme, indolora y evolución insidiosa, que se extendió a regiones adyacentes a la inyección del relleno (Figura 3).

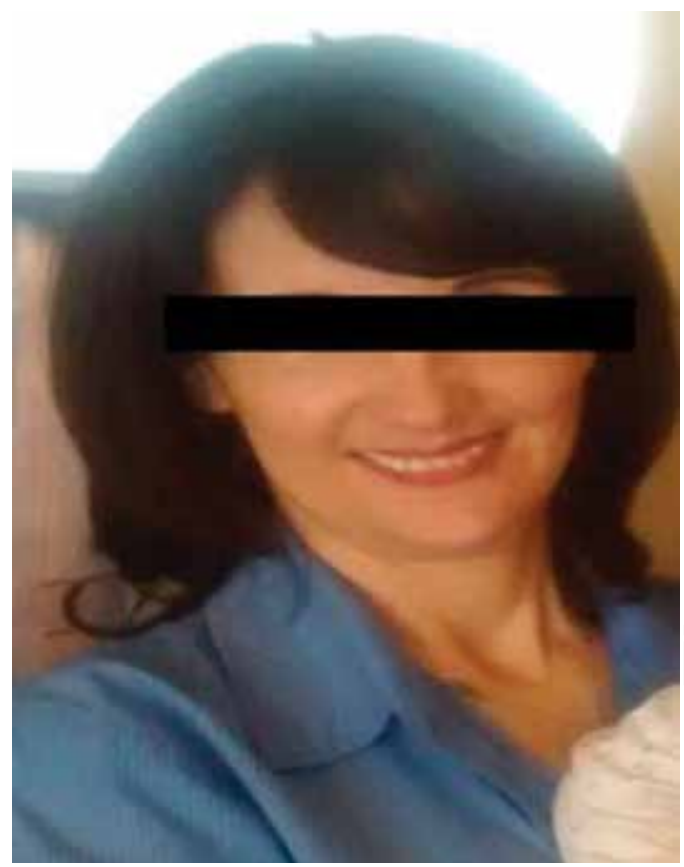

Figura 1: Paciente pretratamiento sin antecedentes de importancia.

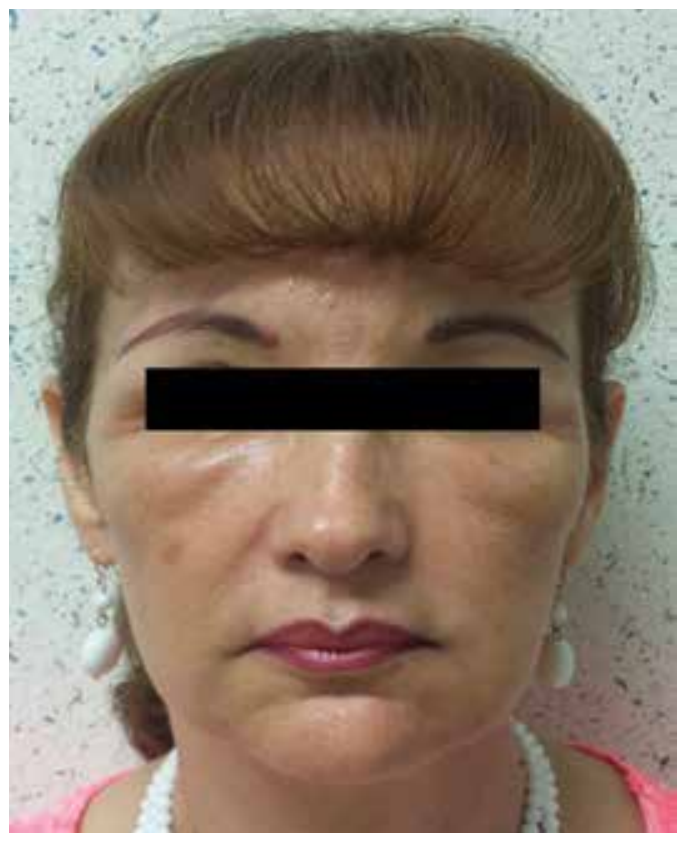

Figura 2: Paciente postratamiento inmediato.

Inició tratamiento con esteroides sistémicos, fármacos antiinflamatorios no esteroideos (AINES) y antihistamínicos. Su evolución fue tórpida, con recaídas del cuadro inflamatorio, por lo que se programó para cirugía en la que se realizó resección del tejido granulomatoso de la zona periorbitaria (Figura 4) y del surco nasogeniano (Figura 5); posteriormente, se continuó el manejo con corticosteroides sistémicos. En un segundo tiempo quirúrgico se realizó ritidectomía para mejorar el aspecto estético; en un futuro, se infiltrará grasa como relleno facial (Figura 6).

\section{DISCUSIÓN}

A pesar de las afirmaciones de fabricantes y de diferentes autores que mencionan que los rellenos no son tóxicos y no inmunogénicos, o que las complicaciones son muy poco frecuentes, los efectos secundarios se producen con todos los compuestos utilizados.

Los biomateriales implantados, inyectados y de contacto con la sangre desencadenan una gran variedad de reacciones adversas, incluyendo inflamación, trombosis y fibrosis excesiva. ${ }^{8,9}$ Estas reacciones adversas se asocian, en general, con la acumulación de un 

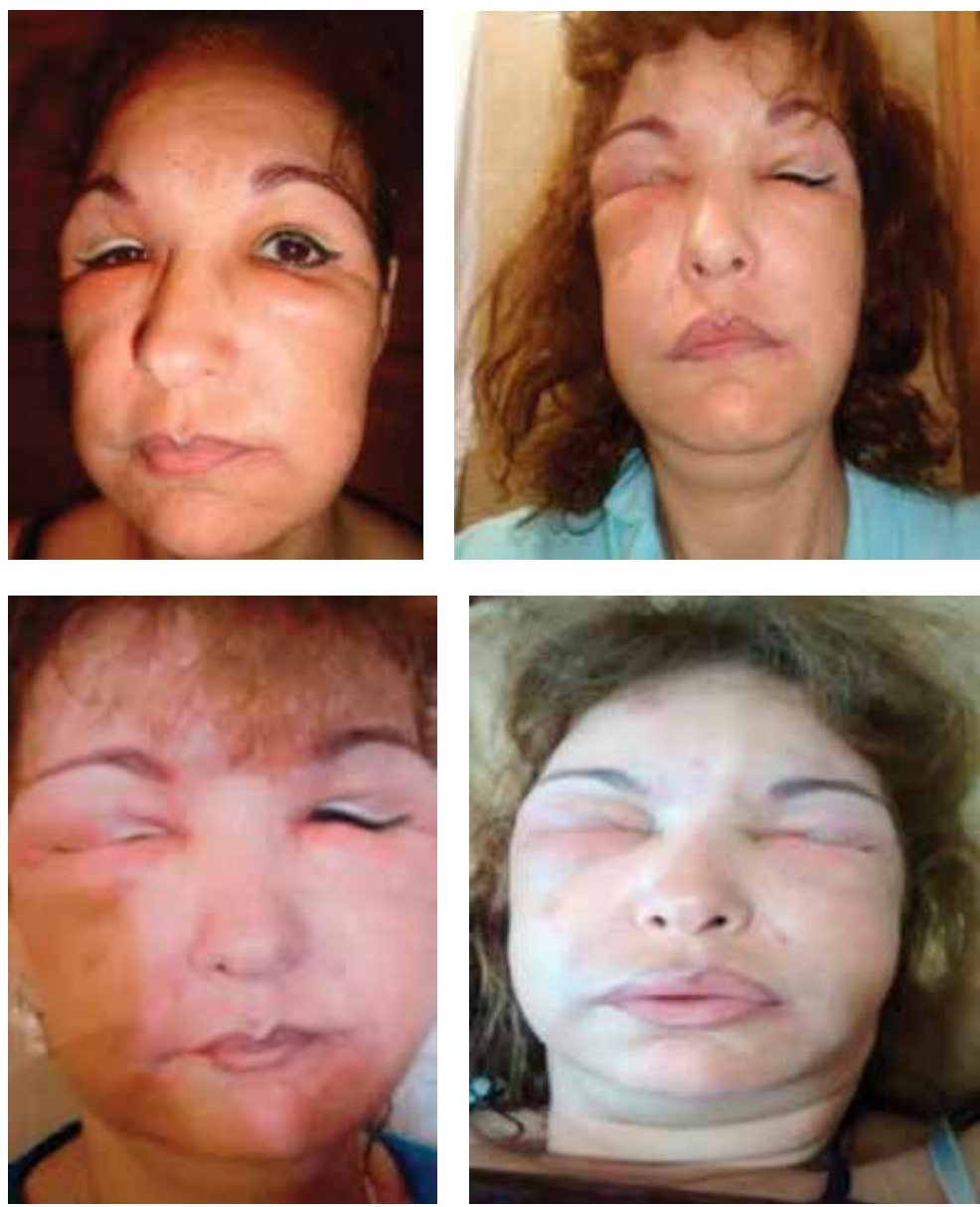

Figura 3: Se observa deformidad debido a una reacción edematosa severa una semana después del tratamiento.
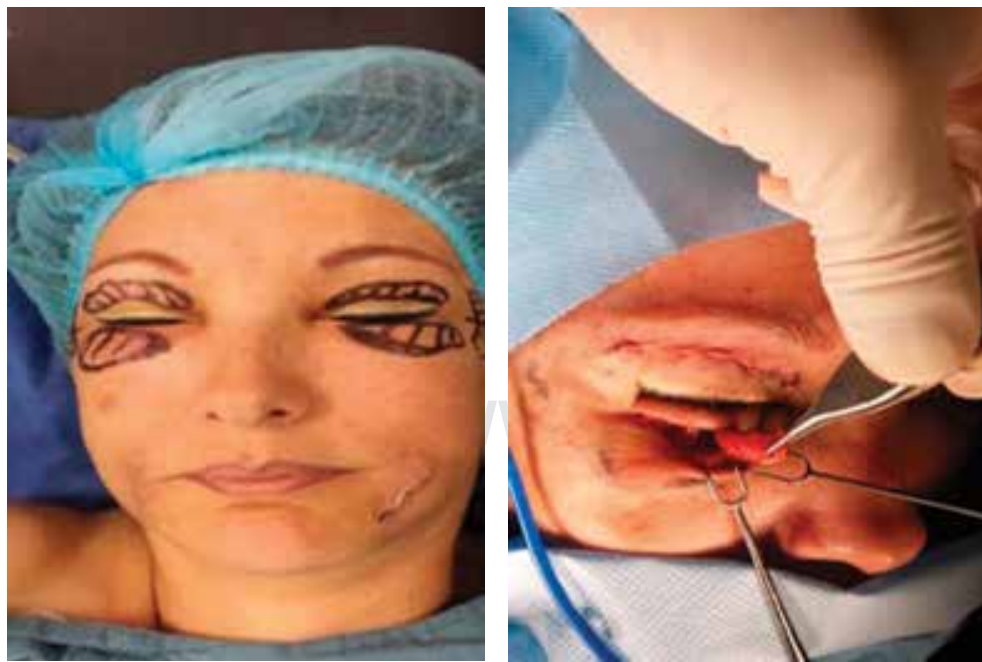

Figura 4: Resección de tejido granulomatoso en zona periorbitaria para su corrección. gran número de células mononucleares. La mayoría de estos efectos adversos parecen tener una base inmunológica; los rellenos actúan más como adyuvantes que como activadores directos de células T en un contexto de predisposición genética, y su tratamiento no ha sido objeto de estudios bien diseñados. El manejo de reacciones agudas y sistémicas es a menudo difícil y requiere terapia antiinflamatoria $y$, ocasionalmente, inmunosupresora. En el caso expuesto, se requirió utilizar corticosteroides sistémicos, fármacos antiinflamatorios no esteroideos (AINES) y retiro del tejido granulomatoso para poder conseguir una evolución óptima de la paciente.

Las reacciones adversas relacionadas con los materiales de relleno comprenden una amplia gama de manifestaciones que pueden aparecer tarde o temprano, y van desde locales hasta sistémicas. Los médicos deben estar conscientes de ellas, ya que el paciente a menudo niega el antecedente de la inyección o desconoce el material empleado.

La selección de un régimen de tratamiento adecuado en los eventos adversos inducidos por el relleno depende del tipo de material, el inicio clínico de las complicaciones, la duración de los granulomas, la localización y el grado. ${ }^{10,11}$ Entre los efectos adversos más graves, se encuentran la isquemia prolongada seguida de compromiso vascular doloroso, necrosis o ulceración, debida a la inyección vascular en la glabela y la formación de tejido granulomatoso. El uso de cánulas romas para la inyección -en lugar de agujas afiladas - reduce notablemente el riesgo de inyecciones intravasculares y de compromiso vascular, evita el uso de más de un relleno al mismo tiempo y en el mismo sitio de inyección $y$, en general, disminuye la tasa de complicaciones.

Las modalidades de tratamiento de los granulomas son diferentes, además de requerir una gran experiencia en la selección apropiada del régimen de manejo, así como la planificación interdisciplinaria y el conocimiento profundo de la farmacología de los agentes. La terapia de primera línea de los granulomas se basa en la inyección intralesional de esteroides cristalinos, incluso a pesar del riesgo de atrofia de la piel. Los agentes inmunomoduladores tópicos y los 
modificadores de la respuesta inmune, tales como imiquimod (Aldara, Zyclara), ungüento de tacrolimus al $0.1 \%$ (Protopic) y crema de pimecrolimus (Elidel) son los más adecuados para el tratamiento inicial de la inflamación granulomatosa superficial. ${ }^{12}$

En los casos resistentes a los esteroides y al tratamiento inmunomodulador, se puede utilizar el 5-fluoruracilo intralesional. En pacientes con granuloma de carga recalcitrante permanente, se puede combinar este tratamiento con la administración oral de alopu-
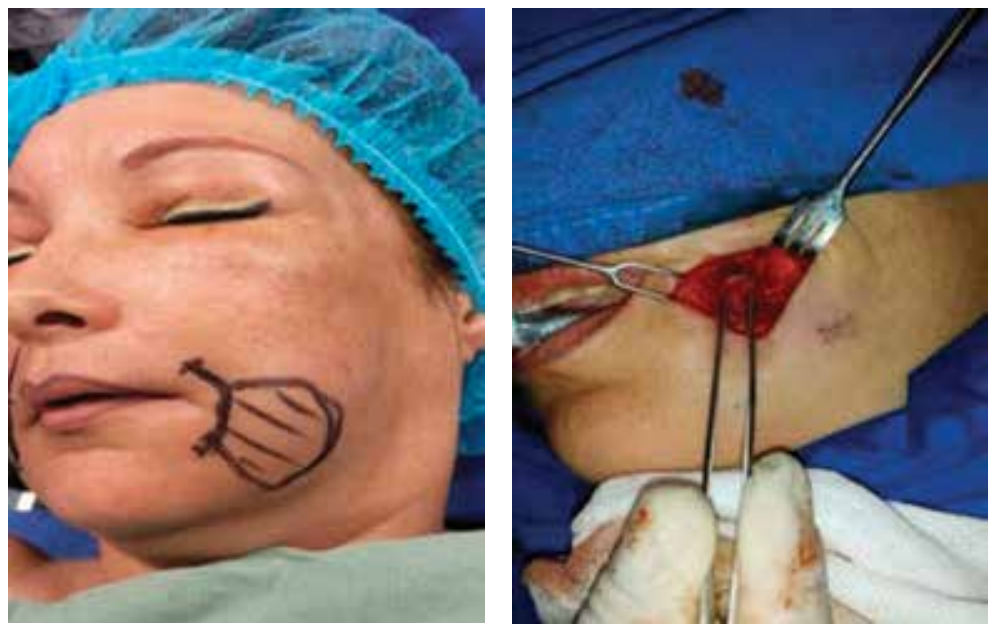

Figura 5: Resección de tejido granulomatoso en surco nasogeniano para su corrección.
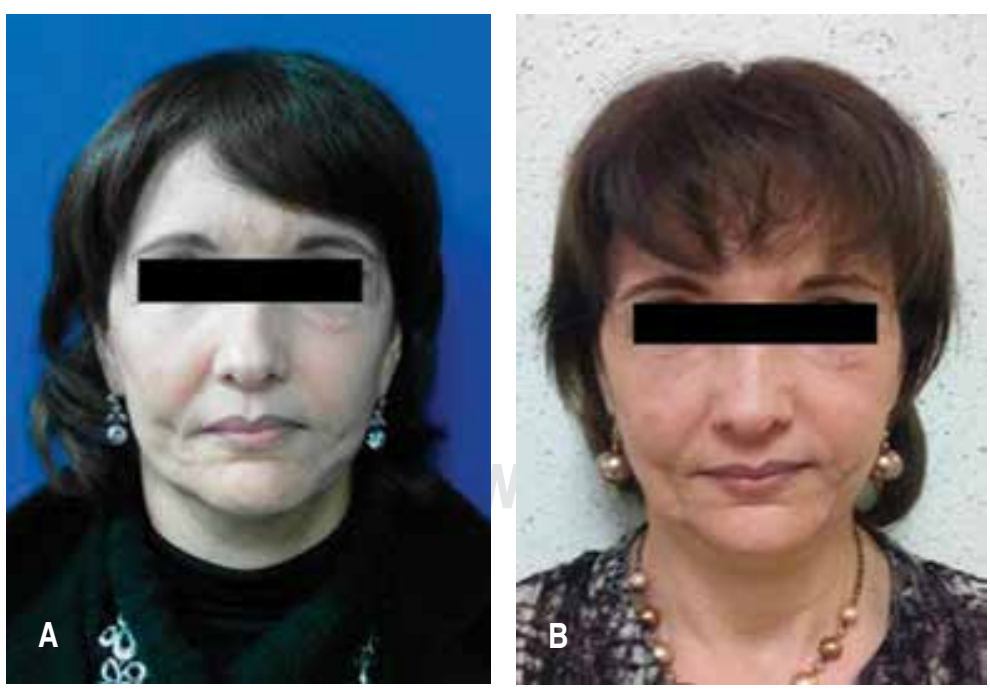

Figura 6: A-B paciente con adecuado resultado posterior a ritidectomía e infiltración grasa. rinol; este tratamiento se prolonga por varios meses, dependiendo del curso clínico. Si se sospecha clínicamente la sobreinfección, puede administrarse por corticosteroides sistémicos en granulomas inflamados agudos, así como antibióticos.

La extirpación quirúrgica de la formación de tejido de granuloma es una opción de último recurso si las otras opciones de tratamiento conservador fallan. El crecimiento del tumor y el aumento del compromiso estético son algunas indicaciones. La tasa de recurrencia es alta y la remoción completa no se puede lograr en la mayoría de los casos.

\section{CONCLUSIONES}

La demanda y uso de procedimientos poco invasivos, como los rellenos faciales, han ido en aumento en los últimos años, por lo que es importante y relevante que el cirujano esté consciente de las posibles complicaciones y reconozca los efectos adversos que surjan en el estudio del paciente. Esto tiene la finalidad de estar preparado para resolver estas situaciones y poder brindar un tratamiento precoz y adecuado, mediante el uso de una buena relación médico-paciente que propicie un proceso quirúrgico con un resultado efectivo.

\section{REFERENCIAS}

1. Alcalá D, Martínez Guerra E. Rellenos Faciales: efectos adversos. Dermatología Cosmética, Médica y Quirúrgica 2013; 11 (1): 36-41.

2. Klein A, Elson M. The history of substances for soft tissue augmentation. Dermatol Surg 2000; 26: 10961105.

3. Haneke E. Adverse effects of fillers and their histopatology. Facial Plast Surg 2014; 30 (6): 599-614.

4. Ballin AC, Brandt F, Cazzaniga A. Dermal fillers: an update. Am J Clin Dermatol 2015; 16 (4): 271-283.

5. Ozturk CN, Li Y, Tung R, Parker L, Piliang MP, Zins JE. Complications following injection of soft-tissue fillers. Aesthet Surg J 2013; 33 (6): 862-877.

6. Wagner R, Fakhro A, Cox J, Izaddoost S. Etiology, prevention, and management of infectious complications of dermal fillers. Sem Plast Surg 2016; 30 (2): 83-86.

7. Funt D, Pavicic T. Dermal fillers in aesthetics: an overview of adverse events and treatment approaches. Clin Cosmet Invest Dermatol 2013; 6: 295-316.

8. Poveda R, Bagán J, Murillo J, Jimenez Y. Reacción granulomatosa facial por rellenos cosméticos inyectados: presentación de cinco casos. Med Oral Patol Oral Cir Bucal 2006; 11: E1-E5. 
9. Lee JM, Kim YJ. Foreign Body Granulomas after the use of dermal fillers: pathophysiology, clinical appearance, histologic features, and treatment. Arch Plast Surg 2015; 42 (2): 232-239.

10. Bigata X, Ribera M, Bielsa I, Ferrandiz C. Adverse granulomatous reaction after cosmetic dermal silicone injection. Dermatol Surg 2001; 27 (2): 198200.

11. Christensen L, Breiting V, Janssen M, Vuust J, Hodgall E. Adverse reactions to injectable soft tissue permanent fillers. Aesthet Plast Surg 2005; 29: 34-48.
12. Baumann L, Halem M. Lip silicone granulomatous foreign body reaction treated with aldara (imiquimod 5\%). Dermatol Surg 2003; 29 (4): 429-432.

Correspondencia:

Dr. Mario Israel Ortega Ascanio

Av. Adrián Muguerza Núm. 115-309,

Col. Brisas, 25210,

Saltillo, Coahuila, México.

Teléfono: 8444851190

E-mail: drisrael74@gmail.com 